\title{
Good to Know
}

\section{Carolle Laure Kpoumie}

Drug Safety Physician- MSL- Medical Expert- Medical Information Medical Research-Medical Writer and Reviewer- Medical Practitionner, Cameroon.

*Corresponding author: Carolle Laure Kpoumie, Drug Safety Physician- MSL- Medical ExpertMedical Information Medical Research-Medical Writer and Reviewer- Medical Practitionner, Cameroon.

Received date: March 13, 2020; Accepted date: April 15, 2020; published date: May o5, 2020

Citation: Carolle Laure Kpoumie, Galenic drugs: plunge into the heart of the secrets of the forms and effects of the medicament. J Clinical Research and Reports, 4(2); DOI:10.31579/2690-1919/o76

Copyright: (C) 2020 Carolle Laure Kpoumie. This is an open access article distributed under the Creative Commons Attribution License, which permits unrestricted use, distribution, and reproduction in any medium, provided the original work is properly cited.

Good practice of taking Medications daily: general view of Paracetamol; Ibuprofen; Aspirin

Always read the leaflet and do not throw it away, stay with the leaflet and its product, if in doubt ask for advice

If any side effects occur, make an appointment directly with your doctor

The most commonly encountered context remains relief of symptomatology usually pain, which involved in the majority of cases - paracetamol, ibuprofen, aspirin.

What do you know about paracetamol?

- Paracetamol is an analgesic (relieves pain)

- Paracetamol is an antipyretic (lowers fever)

- Paracetamol is hepatotoxic (so if you've been told that you have liver disease, so do not take it, or try to stick to the dose because overdose may damage more your liver, and this overdose would damage the same time even if there is no history of a liver problem

- Paracetamol, dosage, always respect it, every 4 hours between taking and even 6 hours in children

- Paracetamol, in self-medication, as analgesic, beyond 5 days of pain, and as antipyretic after 3 days of fever or in case of worsening of your symptomatology, contact your doctor immediately 
- Paracetamol and other medications, make sure that the other medicines you are taking at the same time do not contain paracetamol because of the risk of overdose with the consequences that result - So always check the information on the composition of medicines on the package leaflet or the box of medicines.

\section{What about ibuprofen?}

Ibuprofen was derived from propanoic acid discovered by researchers in the 1960 .

Ibuprofen is a NSAID (nonsteroidal anti-inflammatory drug) generally used for the relief of symptoms of arthritis, fever, primary dysmenorrhea (or menstrual pain) but also as an analgesic (pain reduction) Ibuprofen also has an antiplatelet effect: it protects against the formation of blood clots even if it has a lesser effect than aspirin.

- Ibuprofen is not recommended for Castro duodenal ulcer, gastrointestinal bleeding, allergy and / or asthma, in case of heart disease, kidneys, liver, in elderly patients

- ibuprofen, respect the 6 hour interval between shots

- Ibuprofen, avoid other medicines containing an anti inflammatory or Aspirin

- Some women may have a fertility / infertility problem after long-term use of ibuprofen, a side effect that disappears when you stop taking ibuprofen.

- Ibuprofen has as side effects Diarrhea, nausea, vomiting, dyspepsia (abdominal pain, bloating, indigestion), stomach pain, intestinal pain, hematemesis (vomiting of blood)

Therefore, for greater caution, always read your package leaflet and make sure that the concomitant medications do not contain anti-inflammatory and / or aspirin.

What to know about aspirin?

- Aspirin (acetylsalicylic acid) is an anti-inflammatory analgesic (reduces pain, and antipyretic (lowers fever)

- -respect 4 hours between the shots

- Like paracetamol, ibuprofen, beyond 5 days of pain and 3 days of fever or aggravation of its symptoms, stop the anti-inflammatory and go to medical consultation.

- Aspirin is not a trivial drug. Non-compliance with the dosage or any overdose or prolonged intake is responsible for serious complications.

- Avoid taking it or seek the advice of your doctor in case of a history of stomach ulcer and duodenum, kidney failure, G6PD deficiency, asthma, gout, women with an intrauterine device (STERILET), after tooth extraction, operation or heavy periods, because it increases the risk of bleeding.

- Avoid its use in children with chickenpox or fever caused by a virus (influenza), as there is a risk of REYE syndrome (rare but fatal that is characterized by disturbances of consciousness and vomiting)

- It would be better to use paracetamol or seek the advice of the Doctor. 